\title{
Demonstration of an Open Source Framework for Qualitative Evaluation of CBIR Systems*
}

\author{
Paula Gomez Duran, \\ Eva Mohedano, Kevin McGuinness \\ Insight Center for Data Analytics Dublin \\ City University \\ Dublin, Ireland
}

\author{
Xavier Giró-i-Nieto \\ Image Processing Group \\ Universitat Politecnica de Catalunya \\ Barcelona, Catalonia/Spain \\ xavier.giro@upc.edu
}

\author{
Noel E. O’Connor \\ Insight Center for Data Analytics \\ Dublin City University \\ Dublin, Ireland
}

\begin{abstract}
Evaluating image retrieval systems in a quantitative way, for example by computing measures like mean average precision, allows for objective comparisons with a ground-truth. However, in cases where ground-truth is not available, the only alternative is to collect feedback from a user. Thus, qualitative assessments become important to better understand how the system works. Visualizing the results could be, in some scenarios, the only way to evaluate the results obtained and also the only opportunity to identify that a system is failing. This necessitates developing a User Interface (UI) for a Content Based Image Retrieval (CBIR) system that allows visualization of results and improvement via capturing user relevance feedback. A well-designed UI facilitates understanding of the performance of the system, both in cases where it works well and perhaps more importantly those which highlight the need for improvement. Our open-source system implements three components to facilitate researchers to quickly develop these capabilities for their retrieval engine. We present: a web-based user interface to visualize retrieval results and collect user annotations; a server that simplifies connection with any underlying CBIR system; and a server that manages the search engine data. The software itself is described in a separate submission to the ACM MM Open Source Software Competition.
\end{abstract}

\section{KEYWORDS}

ACM proceedings, $\mathrm{ETEX}$, Visualization tool, image retrieval, visual searching, relevance feedback, annotation tool

\section{ACM Reference format:}

Paula Gomez Duran, Eva Mohedano, Kevin McGuinness, Xavier Giró-iNieto, and Noel E. O'Connor. 2018. Demonstration of an Open Source Framework for Qualitative Evaluation of CBIR Systems. In Proceedings of 2018 ACM Multimedia Conference, Seoul, Republic of Korea, October 22-26, 2018 (MM '18), ACM, New York, NY. 2 pages.

DOI: http://dx.doi.org/10.1145//3240508.3241395

*Produces the permission block, and copyright information

Permission to make digital or hard copies of part or all of this work for personal or classroom use is granted without fee provided that copies are not made or distributed for profit or commercial advantage and that copies bear this notice and the full citation on the first page. Copyrights for third-party components of this work must be honored. For all other uses, contact the Owner/Author.

MM'18, October 22-26, 2018, Seoul, Republic of Korea

(c) 2018 ACM. 978-1-4503-5665-7/18/10.

DOI: http://dx.doi.org/10.1145/3240508.3241395

\section{INTRODUCTION}

The evaluation of most CBIR systems (e.g. $[1,3,4]$ ) is based on quantitative assessment using an appropriate metric, such as mean average precision, to allow for an objective comparison between different approaches. However, qualitative assessments are often equally important, particularly from the perspective of a researcher/developing seeking to improve the underlying models and representations. Qualitative evaluation allows for exploration of success and failure cases to better understand the characteristics of the system and thus how it might be improved.

In this submission, we demonstrate an open source software framework that we have designed to facilitate researchers to qualitatively evaluate their CBIR systems. Our work implements a user interface capable of visualizing results with annotation functionality for relevance feedback. Our software is simple to use and easily adaptable to any CBIR system. It is fully open source, and so available free of change, and extensible to new functional requirements in the future. Very often developers of CBIR systems have to implement a front-end system to better understand how to improve their systems. Our framework is designed to allow them to quickly and easily integrate a front-end UI. The software itself, how to run it, and how to integrate a CBIR system, is described in detail in a separate submission to the ACM Multimedia Open Source Software Competition.

\section{SYSTEM OVERVIEW}

Our system is based on a web application that is able to display the similarity ranking of images retrieved for a given query and, optionally, collect user annotations in order to improve the results. The design of the system consists of a UI that is connected with the CBIR engine through a server that aims to ease the integration of any image retrieval system.

In the demonstration, examples of how to compute rankings will be shown in order to showcase all the different functionalities of the system. The CBIR system used within the framework for this purpose is an adapted version of the CBIR engine ${ }^{1}$ described in [2]. A video is also provided that illustrates typical usage of the framework ${ }^{2}$.

There are three different ways of introducing an input image into the system as shown in figure 1: by introducing its URL, by uploading it from a file or by just selecting any of the example

\footnotetext{
${ }^{1}$ https://bitbucket.org/emohe/python-server-salbow/

${ }^{2}$ https://youtu.be/HOEQi3CfiTc
} 
queries displayed in any dataset. The framework has three main modes of operation: explorer mode, annotation mode and query expansion mode.

\section{- Explorer mode}

Explorer mode can be used to get to know datasets in a dynamic and interactive way. This is achieved by navigating through the database via "clickable" images within rankings list facilitating iterative querying, as shown Figure 1.

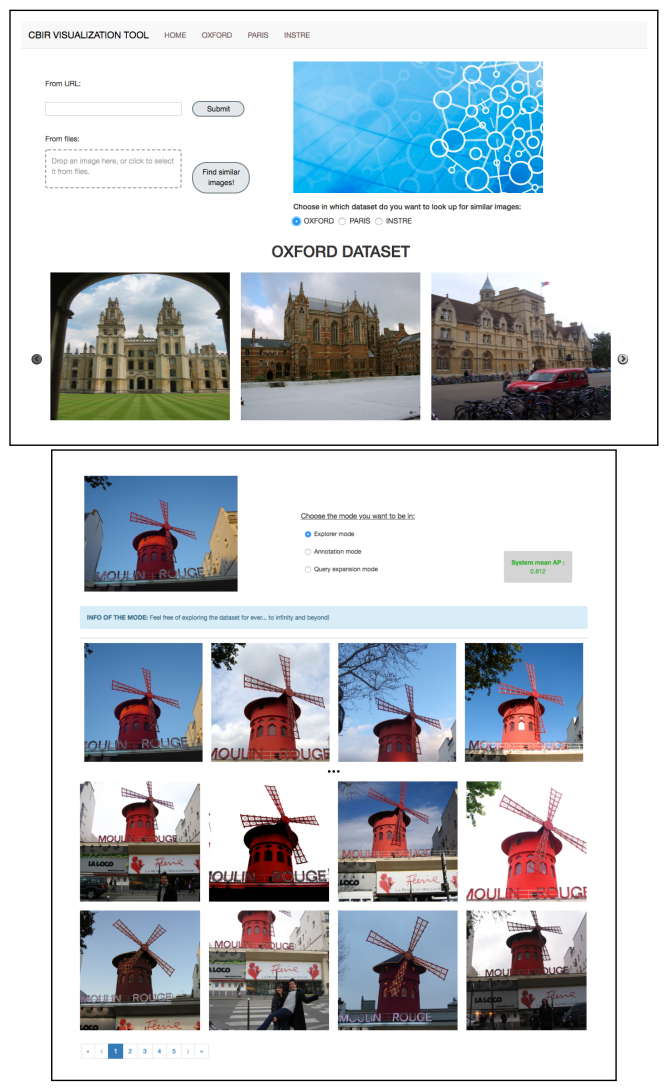

Figure 1: Figure showing the three options to input images in the system: url of an image, image from file or image example of a dataset.

\section{- Query expansion vs annotation mode}

The query expansion and annotation modes are provided to allow investigation of CBIR performance in two different ways. The most appropriate mode to use depends upon the average precision (AP) of a given query. Experimental results show that some rankings improve in accuracy by annotating positive images through the "query expansion" mode. Indeed, this approach will invariably improve performance when the initial AP of the query is very low, since there is a lot of scope for improvement.

However, it can also be shown that the AP of a query can significantly improve when providing negative annotations to the system through the annotation mode. This is due to the fact that negative annotations provide information that allows discarding certain image classes, thus reducing the failure options more directly. Furthermore, this is also useful when refining a query, e.g. to specify one of multiple objects appearing in the query, as can be observed in Figure 2.

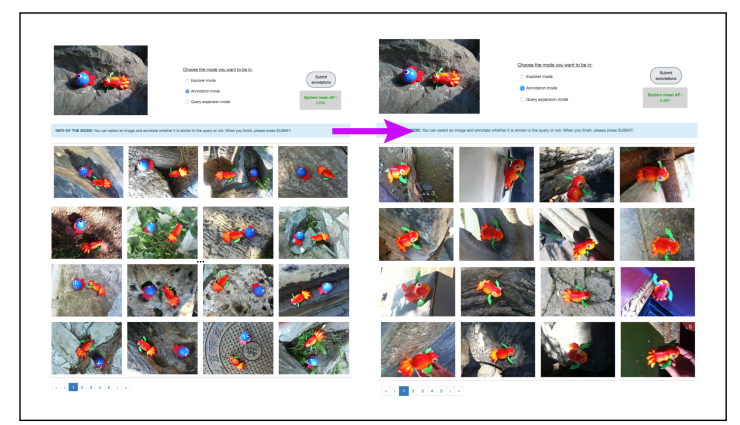

Figure 2: Illustration of the possible use of the annotation tool: in this example, the user used the annotations to select one of the two objects that appeared in the initial query.

\section{CONCLUSION}

In conclusion, we provide an open source software framework that can be used to qualitatively evaluate and improve the performance of a CBIR system. Specifically, as an example of the potential benefits, we show how the system we propose can significantly improve CBIR performance through the provision of negative annotations while, by using only the positive ones, it will help the system to be more precise without significant changes in results. Of course, this implies that "annotation mode" should be regulated by credentials or by logging in a private session in order to have a control of good practices when collecting feedback so as not to negatively impact performance. In contrast, the "query expansion" mode could be used to experiment freely with the system without consequence, as it effectively corresponds to just calculating the mean of the selected image descriptors.

\section{ACKNOWLEDGMENTS}

This publication has emanated from research conducted with the financial support of Science Foundation Ireland (SFI) under grant number SFI/12/RC/2289 and SFI/15/SIRG/3283, and partially supported by the Spanish Ministry of Economy and Competitivity and the European Regional Development Fund (ERDF) under contract TEC2016-75976-R.

\section{REFERENCES}

[1] Yannis Kalantidis, Clayton Mellina, and Simon Osindero. 2016. Cross-Dimensional Weighting for Aggregated Deep Convolutional Features. In ECCV Workshops.

[2] Eva Mohedano, Kevin McGuinness, Xavier Giro-i Nieto, and Noel E O'Connor. 2017. Saliency Weighted Convolutional Features for Instance Search. arXiv preprint arXiv:1711.10795 (2017).

[3] Eva Mohedano, Amaia Salvador, Kevin McGuinness, Ferran Marqués, Noel E. O'Connor, and Xavier Giró. 2016. Bags of Local Convolutional Features for Scalable Instance Search. In ICMR.

[4] Giorgos Tolias, Ronan Sicre, and Hervé Jégou. 2015. Particular object retrieval with integral max-pooling of CNN activations. CoRR abs/1511.05879 (2015). 